\title{
Extraction Procedures and GCxGC-TOFMS Determination of Fatty Acids (FAs) in Cyanobacteria Cultures and the Effect of Growth Media Iron Concentration Variation on Cellular FAs Composition
}

\author{
Kessy F Kilulya ${ }^{1}$, Bhekie B Mamba ${ }^{2}$ and Titus AM Msagati ${ }^{2^{*}}$ \\ ${ }^{1}$ University of Dar es Salaam: Chemistry Department, PO Box 35061, Dar es Salaam, Tanzania
}

${ }^{2}$ University of South Africa, College of Science Engineering and Technology, Nanotechnology and Water Sustainability Research Unit, UNISA Science Campus, P.O. Box 392 UNISA 0003, Florida, 1709 Johannesburg, South Africa

*Corresponding author: Msagati TAM, University of South Africa, College of Science Engineering and Technology, Nanotechnology and Water Sustainability Research Unit UNISA Science Campus, P.O. Box 392 UNISA 0003, Florida, 1709 Johannesburg, South Africa, Tel: +27 11 670 9482; Email: msagatam@unisa.ac.za

Received date: January 15, 2015, Accepted date: March 19, 2015, Published date: March 26, 2015

Copyright: ( 2015 Kilulya KF, et al. This is an open-access article distributed under the terms of the Creative Commons Attribution License, which permits unrestricted use, distribution, and reproduction in any medium, provided the original author and source are credited.

\begin{abstract}
Cyanobacteria biomass obtained from freshwater bodies is known to contain a large amount of fatty acids. Thus, the current need to produce large amount of lipids for different application has identified cyanobacteria as one of the important sources of fatty acids. However, the production of fatty acids and the characteristics of its composition is a function of nutrients. This paper therefore, reports on the efficient extraction procedures of fatty acids from cyanobacteria biomass using ionic liquid solvent and organic solvent under ultrasonic solid liquid extraction technique and the effect of the variation of iron concentration in the growth media on the fatty acids composition of cyanobacteria biomass. The determination of fatty acids composition was performed using gas chromatography coupled to a time-of-flight mass spectrometer (GCxGC-TOFMS). The obtained fatty acids composition was found to be dominated by tetradecanoic acid (C14:0), hexadecanoic acid (C16:0), 7-hexadecenoic acid (C16:1), pentadecanoic acid (C15:0), 6,9,12,15-octadecatetraenoic acid (C18:4), $\mathrm{y}-6,9,12$-octadecatrienoic acid ( $\mathrm{\gamma}$-C18:3), 9,12,15-octadecatrienoic acid (C18:3), 9,12-octadecadienoic acid (C18:2), 9-octadecenoic acid (C18:1) and octadecanoic acid (C18:0). Statistically it was proved that the ionic liquid extraction protocol and that of ultrasonic solid liquid extraction by organic solvent were not significantly different. It was also revealed that the total amount of fatty acids in cultured cyanobacteria increased with decrease in iron concentration in the growth medium.
\end{abstract}

Keywords: Cyanobacteria; Iron concentration; Fatty acids; Gas Chromatography; Time-of-flight; Mass spectrometry; Ionic liquid

\section{Introduction}

Cyanobacteria are a diverse group of photosynthetic organisms found mostly in freshwater bodies [1]. Cyanobacteria, apart from producing different useful metabolites for different applications such as pharmaceuticals, production of fine chemicals, biofertilizers and animal feed [2-5], they are known to produce lipophilic extractives such as fatty acids which are somewhat toxic to aquatic organisms, especially unsaturated fatty acids. They are known to affect fish in freshwater bodies by interfering with the gill basolateral membrane ion-extrusion mechanisms leading to the death of fish $[1,6]$. Thus, the toxicity properties of fatty acids released by cyanobacteria in freshwater bodies to other aquatic organisms have increased the need to characterise the composition of fatty acids in cyanobacteria. Currently the production of biodiesel has increased the importance of lipids and hence the extraction techniques from different sources [7]. Moreover, fatty acids have been identified as essential components of the diet of humans and animals [8]. This fact has highly contributed to the interest of different researchers on the effective techniques to obtain fatty acids from cyanobacteria. Polyunsaturated fatty acids such as 9,12-Octadecadienoic acid (C18:2) and $\gamma$-6,9,12-octadecatrienoic acid $(\gamma-\mathrm{C} 18: 3)$ are known for their human health importance $[1,9]$. Furthermore, the analysis of long chain fatty acids has been so significant for biological and medical investigations [7]. The most prominent analytical instrument for fatty acids analysis is gas chromatography-mass spectrometry (GC-MS) [10]. However, due to the separation problem of fatty acids with similar chemical structures such as fatty acids with a chain of 18 carbon atoms, a more advanced gas chromatographic technique (GCxGC-TOFMS) has been employed in fatty acids and lipids analysis. GCxGC-TOFMS is a powerful analytical technique for the qualitative and quantitative determination of lipid composition. Fatty acids are currently best determined by GCxGC-TOFMS after derivatization to fatty acids methyl esters (FAMEs). The use of deconvolution algorithm of ChromaTOF software of GCxGC-TOFMS enables the separation of mass spectra and identification of FAMEs which some have a tendency of coeluting and bearing similar mass spectra due to their similar chemical structures. Extraction of fatty acids and lipids from the solid samples has generally been achieved by using volatile organic solvents under different extraction techniques reported in the literature. However, due to the nature of algae biomass, and the fact that they contain unusual lipid classes and fatty acids which differ from the ones in higher animals and plants [7], it is important to investigate a suitable extraction method for lipids and fatty acids from algae biomass. Among the techniques which are reported to be suitable, ultrasonic solid liquid extraction techniques has been found to be more effective as well as time effective [11]. Lipids and fatty acids in particular can be effectively extracted using polar organic solvents which are able to extract a larger range of organic compounds from biomass samples mostly at an elevated temperature [12]. This is attributed to the fact that at higher temperature the polar solvent decreases its polarity to allow it to capture compounds ranging from semi-polar to non-polar 
Citation: Kilulya KF, Bhekie B Mamba BB, Msagati TA (2015) Extraction Procedures and GCxGC-TOFMS Determination of Fatty Acids (FAs) in Cyanobacteria Cultures and the Effect of Growth Media Iron Concentration Variation on Cellular FAs Composition. J Environ Anal Toxicol S7: S7-009. doi:10.4172/2161-0525.S7-009

Page 2 of 6

$[11,13,14]$.The mixture of chloroform and methanol has been reported to be the most effective solvent composition for extraction of lipids from cyanobacteria biomass. However, due to the environmental issues the newly emerging ionic liquids have been utilized in the extraction and analyses of different organic compounds from different matrices [15-18]. Based on the stipulated importance and effects of fatty acids, the characterization of its composition obtained from different sources is vital. Thus, this paper reports on the effect of iron concentration as one of the macro nutrients on the qualitative and quantitative composition of fatty acids produced by cyanobacteria. The study also presents the comparison of the extraction procedures between the ionic liquid based and organic solvent based procedure and their determination using GCxGC-TOFMS.

\section{Materials and Methods}

\section{Sample collection and pretreatment}

Cyanobacteria samples were collected from Vaal Dam in South Africa. Vaal Dam which is situated in Vaal River supplies water to Gauteng Province and other areas. Samples were collected under the coordination of Rand water treatment Plant Company. After collection samples were washed and freeze dried prior to the extraction. The collected samples were filtered and washed to remove any contaminants followed by freeze drying and frozen until analysis

\section{Culturing of cyanobacteria}

Cyanobacteria samples which were initially collected from Vaal Dam in South Africa were sub-cultured in the laboratory at a controlled supply of iron sulphate. A stock solution of GBG11 culture medium used for growth of cyanobacteria was composed of $\mathrm{NaNO}_{3}$ $15 \mathrm{~g} / \mathrm{L}, \mathrm{K}_{2} \mathrm{HPO}_{4} \cdot 3 \mathrm{H}_{2} \mathrm{O} 4.93 \mathrm{~g} / \mathrm{L}, \mathrm{MgSO}_{4} .7 \mathrm{H}_{2} \mathrm{O} 7.50 \mathrm{~g} / \mathrm{L}, \mathrm{CaCl}_{2} .2 \mathrm{H}_{2} \mathrm{O}$ $3.60 \mathrm{~g} / \mathrm{L}, \mathrm{Na}_{2} \mathrm{SiO}_{3} 10.0 \mathrm{~g} / \mathrm{L}, \mathrm{Na}_{2} \mathrm{CO}_{3} 2.0 \mathrm{~g} / \mathrm{L}$, EDTA $0.10 \mathrm{~g} / \mathrm{L}$, citric acid $1.20 \mathrm{~g} / \mathrm{L}, \mathrm{FeSO}_{4} .7 \mathrm{H}_{2} \mathrm{O} 1.10 \mathrm{~g} / \mathrm{L}$ and micro-elements comprising $\mathrm{H}_{3} \mathrm{BO}_{4}$ $2.86 \mathrm{~g} / \mathrm{L}, \quad \mathrm{MnCl}_{2} .4 \mathrm{H}_{2} \mathrm{O} \quad 1.18 \mathrm{~g} / \mathrm{L}, \quad \mathrm{ZnSO}_{4} .7 \mathrm{H}_{2} \mathrm{O} \quad 0.222 \mathrm{~g} / \mathrm{L}$, $\mathrm{NaMoO}_{4} \cdot 2 \mathrm{H}_{2} \mathrm{O} \quad 0.313 \mathrm{~g} / \mathrm{L}, \quad \mathrm{Co}\left(\mathrm{NO}_{3}\right)_{2} \cdot 6 \mathrm{H}_{2} \mathrm{O} \quad 0.0494 \quad \mathrm{~g} / \mathrm{L}$ and $\mathrm{CuSO}_{4} \cdot 5 \mathrm{H}_{2} \mathrm{O} 0.079 \mathrm{~g} / \mathrm{L}$. This stock solution was used to make a mixture of GBG11 growth medium by taking $10 \mathrm{~mL}$ of the macronutrients elements and $1 \mathrm{~mL}$ of a mixture of micro elements making it up to $1 \mathrm{~L}$. The solution was sterilised in autoclave at $121^{\circ} \mathrm{C}$, cooled to room temperature then $150 \mathrm{~mL}$ of the solution was used for growth of cyanobacteria in a $500 \mathrm{~mL}$ Erlenmeyer flask, with a cotton wool stopper. The growth took place at a light intensity of $100 \mu \mathrm{E} / \mathrm{m}^{2} / \mathrm{g}$ incubated in a water bath maintained at a temperature of $25 \mathrm{C}$ at a $\mathrm{pH}$ of 8.30. The harvest of the cultured cyanobacteria was done after every 21 days per each concentration of iron which was added as $\mathrm{FeSO}_{4}$. The harvested samples were filtered and washed to remove any contaminants followed by freeze drying and frozen until analysis.

\section{Chemical reagents and standards}

Hexane, acetone, methanol, chloroform, anhydrous sodium sulphate, Hydrochloric acid, methyl tetradecanoate, methyl hexadecanoate, methyl linoleate, methyl oleate, methyl linolenate, methyl octadecanoate were of analytical grade and they were all purchased from Sigma Aldrich (St Louis, Mo, USA).

\section{Analytical Procedures and Methods}

\section{Ultrasonic solid liquid extraction}

Ultrasonic solid liquid extraction (USLE) technique was used for extraction of lipids by using $20 \mathrm{~mL}$ of the mixture of chloroform and methanol 1:1 v/v. The extraction was carried out by first incubating the sample mixture in water bath for $30 \mathrm{~min}$ at $60^{\circ} \mathrm{C}$ then sonicated at the same temperature for 1 hour. The extracts were filtered using PTFE filters of $0.2 \mu \mathrm{m}$ pore sizes. Solvent was then evaporated to dryness and the extracts re-dissolved in $1 \mathrm{~mL}$ of acetone and $0.5 \mathrm{~mL}$ of $3 \mathrm{M}$ methanolic $\mathrm{HCL}$ and heated at $60^{\circ} \mathrm{C}$ for $1 \mathrm{~h}$ in a thermostated water bath for derivatization. Thereafter the samples were cooled at room temperature followed by the addition of $1 \mathrm{~mL}$ of water to obtain phase separation and extracted using $1 \mathrm{~mL}$ x 3 of hexane. Extracts were mixed and dried using anhydrous sodium sulphate followed by solvent evaporation to dryness. Then, the extracts; Fatty acids methyl esters (FAMEs) were dissolved in $1 \mathrm{~mL}$ of HPLC grade hexane, filtered using PTFE disc filters of $0.2 \mu \mathrm{m}$ pore sizes for GCxGC-TOFMS analysis. All samples were analysed in duplicate.

\section{Ionic liquid extraction procedure}

About $0.1 \mathrm{~g}$ of freeze dried algae biomass was weighed in duplicate and dissolved in about $2.85 \mathrm{~g}$ of molten ionic liquid at $90^{\circ} \mathrm{C}$ in which the dissolution took about $3 \mathrm{~min}$ to form a non-viscous uniform solution of $5 \%$ algae biomass. About $5 \mathrm{~mL}$ of methanol was added to form slurry followed by the addition of $4 \mathrm{~mL}$ of $3 \mathrm{M}$ methanolic hydrochloric acid and the slurry was homogenized by vortexing. The mixture was then heated for $1 \mathrm{~h}$ at $60^{\circ} \mathrm{C}$ in a thermostated water bath for derivatization followed by cooling at room temperature. About 4 $\mathrm{mL}$ of distilled water was added for phase separation followed by extraction of Fatty acids methyl esters (FAMEs) with $4 \mathrm{~mL}$ of hexane $\times$ 2 under vigorous vortexing. Extracts were then mixed and dried using anhydrous sodium sulphate and solvent evaporated to dryness. The extracts; FAMEs were dissolved in $1 \mathrm{~mL}$ of HPLC grade hexane, filtered using PTFE disc filters with a pore size of $0.2 \mu \mathrm{m}$, for GCxGCTOFMS analysis.

\section{GCxGC-TOFMS conditions}

The analysis of both standards and samples were carried out using GCxGC-TOFMS (Pegasus 4D, LECO Corporation). Helium was used as a carrier gas whereas nitrogen, compressed air and liquid nitrogen used for the operation of the quad-jet thermal modulator. The sample injector temperature was set at $280^{\circ} \mathrm{C}$, and samples were injected at a volume of $1 \mu \mathrm{L}$ with splitless mode. The flow of carrier gas was set at a rate of $1 \mathrm{~mL} / \mathrm{min}$. The GCxGC column set comprised of a $30 \mathrm{~m}$, RXI-5Sil MS (0.25 mm internal diameter, $0.25 \mu \mathrm{m}$ stationary film thickness) for the first while the second column was $1.36 \mathrm{~m}$, RTX-200 (0.18 $\mathrm{mm}$ internal diameter, $0.18 \mu \mathrm{m}$ stationary film thickness). Temperature programme on the first column oven was $80^{\circ} \mathrm{C}$ held for 1 minute, then increased to $290^{\circ} \mathrm{C}$ at a ramping rate of $10^{\circ} \mathrm{C} / \mathrm{min}$ and held for 5 minutes. Whereas the second dimension column oven temperature started at $90^{\circ} \mathrm{C}$ held for $1 \mathrm{~min}$. then ramped to $300^{\circ} \mathrm{C}$ at a ramping rate of $10^{\circ} \mathrm{C} / \mathrm{min}$. and held for 5 minutes. The modulator interface was set at $15^{\circ} \mathrm{C}$ above the secondary oven temperature. The transfer line temperature was set at $250^{\circ} \mathrm{C}$ whereas the ion source temperature was set at $240^{\circ} \mathrm{C}$. Electron impact ionization energy was set at $70 \mathrm{eV}$, while the detector voltage was set at $1600 \mathrm{~V}$. The analysis was carried out at mass range of 40-450 amu and acquisition rate was 
Citation: Kilulya KF, Bhekie B Mamba BB, Msagati TA (2015) Extraction Procedures and GCxGC-TOFMS Determination of Fatty Acids (FAs) in Cyanobacteria Cultures and the Effect of Growth Media Iron Concentration Variation on Cellular FAs Composition. J Environ Anal Toxicol S7: S7-009. doi:10.4172/2161-0525.S7-009

Page 3 of 6

100 spectra/second. The concentration calculations of the identified analytes were carried out using Microsoft Excel.

Identification of the analytes was achieved by comparison of the retention times of the analytes and the standards, comparison of mass spectra with those in the MS Library, and on the basis of mass fragmentation pattern of the compounds.

\section{Statistical Data analysis}

The comparison between the performance of ionic liquid based extraction and the conventional volatile organic solvent based extraction was performed using One-Way ANOVA with a significance level of 0.05

\section{Results and Discussion}

\section{Determination of fatty acids from algae biomass by GCxGC- TOFMS}

The qualitative and quantitative determination of fatty acids (determined as fatty acid methyl esters) from cyanobacteria biomass was effectively performed using GCxGC-TOFMS technique in which the identification of compounds was facilitated by the deconvolution algorithm of ChromaTOF software. ChromaTOF software enabled the separation, identification and accurate quantifications of chromatogram peaks of fatty acids methyl esters (FAMEs) which were somehow coeluting as illustrated on Figure 1. Most of the C18s fatty acids isomers do coelute in chromatography due to their structure similarities which only differ by the number and positions of double bonds.

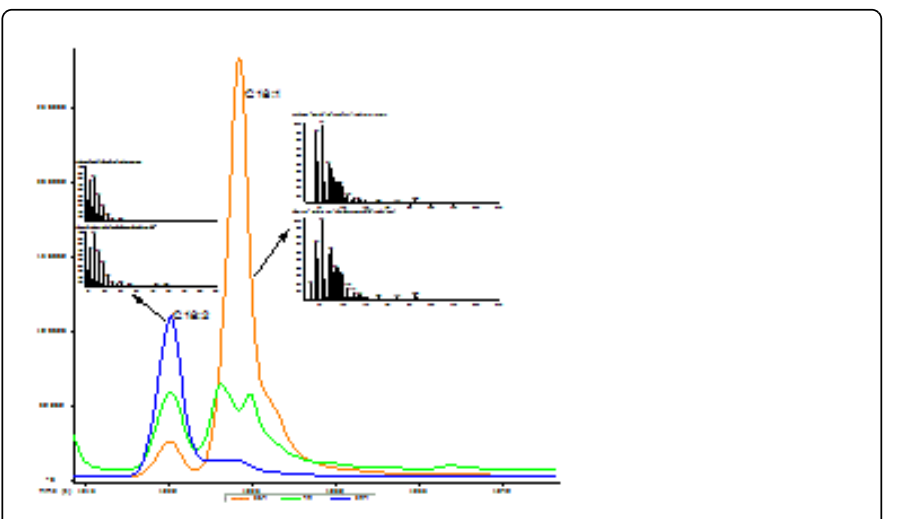

Figure 1: One-dimensional GC-TOFMS mass spectral deconvolution of $\mathrm{C} 18: 2$ and $\mathrm{C} 18: 1$ fatty acids

Moreover, the consideration of analytes peaks in both dimensions of the GCxGC-TOFMS (ie one-dimension (1D) and two-dimension (2D)) chromatography enabled an accurate identification of the compounds (fatty acids methyl esters) as illustrated in Figure 2.

The quantification of identified peaks of fatty acids methyl esters was performed by the use of calibration curve obtained by running different concentrations of mixed standards of fatty acids methyl esters. The mixed standard contained tetradecanoic acid methyl ester, hexadecanoic acid methyl ester, 9,12-octadecadienoic acid methyl ester, octadecanoic acid methyl ester, 9,12,15-octadecatrienoic acid methyl ester, 9-octadecenoic acid methyl ester and Undecanoic acid methyl ester (used as internal standard). The obtained calibration curves had linear coefficient (r2) values ranging between 0.9779 and 0.9978. The determined and quantified fatty acids in the cultured samples at varying amounts with respect to the concentration of iron were: dodecanoic acid (C12:0), tetradecanoic acid (C14:0), 10tetradecenoic acid (C14:1), pentadecanoic acid (C15:0), hexadecanoic acid (C16:0), 7,10-hexadecadienoic acid (C16:2), 7-Hexadecenoic acid (C16:1), heptadecanoic acid (C17:0), 6,9,12,15-octadecatetraenoic acid $(\mathrm{C} 18: 4), \quad \gamma$-6,9,12-octadecatrienoic acid $\quad(\gamma$-C18:3), 9,12,15octadecatrienoic acid (C18:3), 9,12-octadecadienoic acid (C18:2), 9octadecenoic acid (C18:1), octadecanoic acid (C18:0), eicosanoic acid (C20:0), 15-tetracosenoic acid (C24:1), tetracosanoic acid (C24:0), hexacosanoic acid (C26:0) and octacosanoic acid (C28:0).

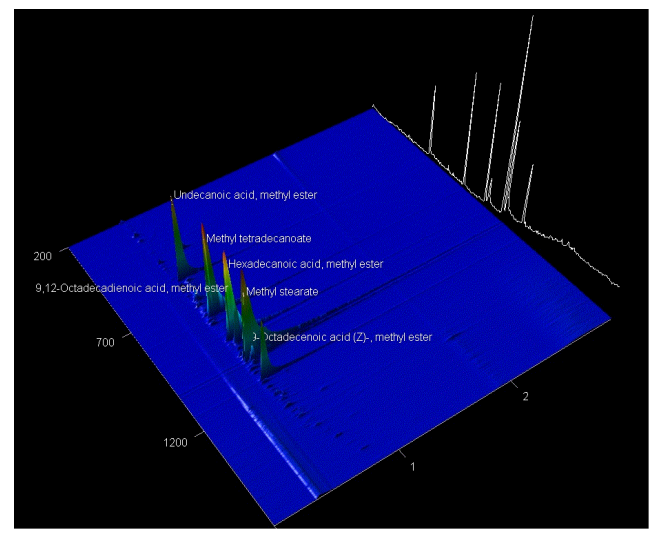

Figure 2: Two dimensional (2D) GCxGC-TOFMS chromatography showing the identification of fatty acids methyl esters standard signals (Undecanoic acid methyl ester was used as an internal standard).

\section{Determination of the effect of Iron concentration on the amount of Fatty Acids}

Determination of the effect of the variation of iron concentration on the amount of fatty acids in the growth medium of cyanobacteria revealed that the total amount of fatty acids in the cultured cyanobacteria decreased with increase in $\mathrm{FeSO} 4$ concentration Figure 3. This can be explained by the importance of iron in the growth of cyanobacteria in water. It has been reported that iron promotes the growth of cyanobacteria in natural waters. Its additions increase photosynthesis and nitrogen fixation by cyanobacteria [19].

It was further observed that the total amount of unsaturated fatty acids decreased with increase in the concentration of $\mathrm{FeSO} 4$ whereas the amount of saturated fatty acids increased with increasing concentration of FeSO4. This can be explained by the fact that under stressful conditions saturated fatty acids are desaturated to produce more unsaturated fatty acids. The unsaturation of fatty acids is used to control the fluidity of cell membranes caused by such stress $[8,20]$. In different stress conditions like $\mathrm{pH}$ stress, temperature stress, and nutrient shortage stress, cyanobacteria tend to produce high amount of toxins [21]. Apart from producing more toxins, the stressful condition leads to the development of a mechanism that sustains their life. One of the mechanisms used to overcome stresses involves desaturation of fatty acids which result on the production of more unsaturated fatty acids. Thus, the trend observed in Figure 3 adheres 
Citation: Kilulya KF, Bhekie B Mamba BB, Msagati TA (2015) Extraction Procedures and GCxGC-TOFMS Determination of Fatty Acids (FAs) in Cyanobacteria Cultures and the Effect of Growth Media Iron Concentration Variation on Cellular FAs Composition. J Environ Anal Toxicol S7: S7-009. doi:10.4172/2161-0525.S7-009

Page 4 of 6

to the fact that under Fe shortage more unsaturated fatty acids were produced. The variations observed in the amount of the components of fatty acids as indicated in Figure 3, shows that unsaturated fatty acids increased with the decrease in the iron concentration whereas the concentration of saturated fatty acids increased with the increase in iron concentration. This indicates that at adequate or higher amount of iron the desaturation of fatty acids is minimal. Generally, it can be noted from Figure 3 that $2.2 \mathrm{mg} / \mathrm{L}$ of FeSO 4 is the optimum concentration as it gives the maximum amount of total fatty acids. The observed trend was then investigated further to find out the contribution of individual fatty acid on the general observed trend by histogram presentation as shown in Figures 4 and 5. For ease comparisons the fatty acids were grouped into saturated and unsaturated components.

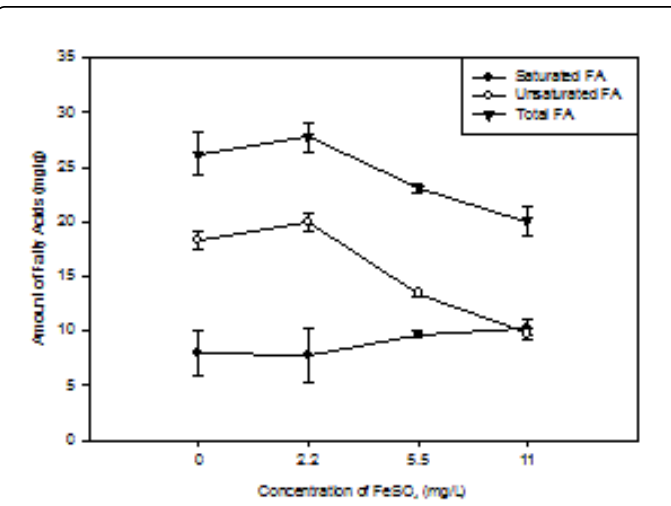

Figure 3: The effect of iron concentration (added as FeSO4) variation on the amount of fatty acids produced by Cyanobacteria

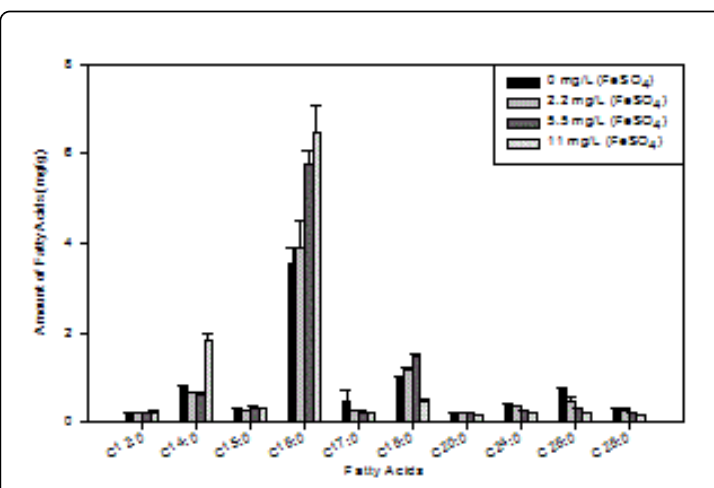

Figure 4: Effect of the variation of iron concentrations on the amount of saturated fatty acids detected in cultured cyanobacteria biomass

Figure 4 shows clearly that the amount of hexadecanoic acid increased significantly with the increase of Fe concentration in the culture media. A slight increasing trend was observed in dodecanoic acid (C12:0), Tetradecanoic acid (C14:0) and pentadecanoic acid (C15:0). However, C14:0 registered its highest amount at the concentration of $11 \mathrm{mg} / \mathrm{L}$ of FeSO 4 indicating favoured production at that concentration. The concentration of octadecanoic acid (C18:0) was found to increase at a range of $0 \mathrm{mg} / \mathrm{L}$ of FeSO 4 to $5.5 \mathrm{mg} / \mathrm{L}$ of FeSO 4 then decreased at the concentration of $11 \mathrm{mg} / \mathrm{L}$ of FeSO4. A slightly decreasing trend in the saturated fatty acids components was realised in the fatty acids with longer carbon chains such as tetracosanoic acid (C24:0), hexacosanoic acid (C26:0) and octacosanoic acid (C28:0).

In the observation of Figure 5, it was found that 7-hexadecenoic acid (C16:1) was increasing with the increase in concentration whereas $\gamma-6,9,12$-octadecatrienoic acid $(\gamma$-C18:3) was very higher at $0 \mathrm{mg} / \mathrm{L}$ of FeSO4 which indicates that it is highly formed during a stressful condition (shortage of iron). The highest amount of 6,9,12,15octadecatetraenoic acid (C18:4) was registered at the concentration of $2.2 \mathrm{mg} / \mathrm{L}$ of $\mathrm{FeSO} 4$, which indicates that its production is influenced by relatively low amount of iron. Thus, the concentration of $2.2 \mathrm{mg} / \mathrm{L}$ of FeSO4 seems to be the optimal amount for the production of C18:4, Figure 5. A general observation from the presented variations is that the type and amount of fatty acids produced by cyanobacteria can be controlled by varying the type and amount of nutrients offered.

\section{Effect of Nutrients on Fatty Acids composition}

Generally the effect of nutrients on the composition of fatty acids in cyanobacteria biomass was determined by comparing the results obtained from cultured cyanobacteria samples against samples which were collected from Vaal Dam. Thus, fatty acids obtained from cultured cyanobacteria biomass differed from the composition of fatty acids in cyanobacteria obtained from Vaal Dam. This could be explained by the differences in nutrient contents. The cultured cyanobacteria were supplied with all the necessary nutrients for their growth whereas the one in the Dam simply depended on the nutrient available in the Dam (natural environment). For instance, 7, 10hexadecadienoic acid (C16:2), 15-tetracosenoic acid (C24:1), tetracosanoic acid (C24:0), hexacosanoic acid (C26:0) and octacosanoic acid (C28:0) were not detected in the cyanobacteria biomass from the Dam samples but detected in the cultured cyanobacteria biomass. On the other hand C19:0 was only detected in cyanobacteria samples from Vaal Dam and not in the cultured samples, Table 1. This means that with the provision of the required nutrients, cyanobacteria biomass produce more amount of fatty acids with longer carbon chain compared to the ones which grow in the natural settings.

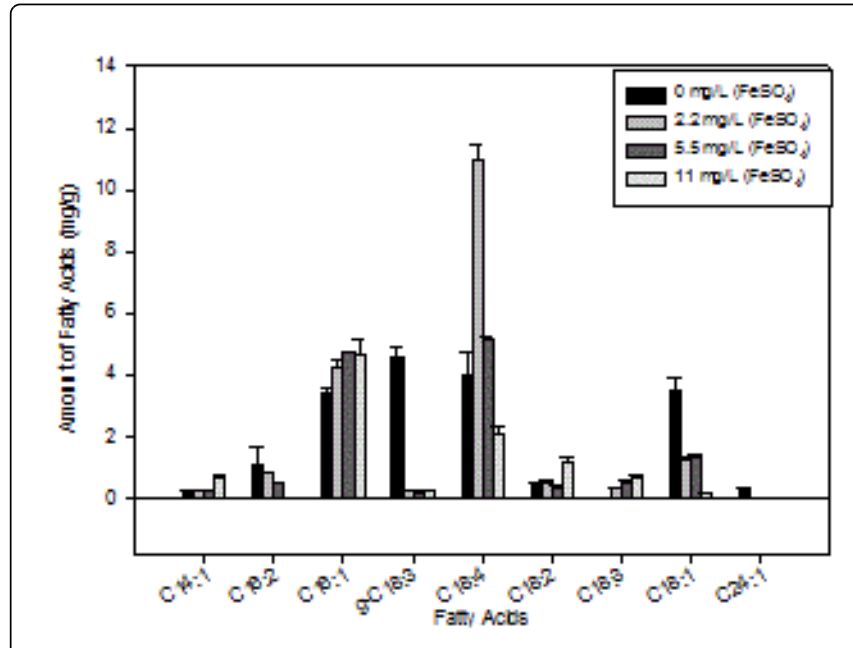

Figure 5: Effect of the variation of iron concentrations on the amount of unsaturated fatty acids detected in cultured cyanobacteria biomass 
Citation: $\quad$ Kilulya KF, Bhekie B Mamba BB, Msagati TA (2015) Extraction Procedures and GCxGC-TOFMS Determination of Fatty Acids (FAs) in Cyanobacteria Cultures and the Effect of Growth Media Iron Concentration Variation on Cellular FAs Composition. J Environ Anal Toxicol S7: S7-009. doi:10.4172/2161-0525.S7-009

Page 5 of 6

\begin{tabular}{|c|c|c|c|c|c|}
\hline \multicolumn{3}{|c|}{ Saturated fatty acids } & \multicolumn{3}{|c|}{ Unsaturated fatty acids } \\
\hline \multicolumn{3}{|c|}{ Mean \pm STDEV } & \multicolumn{3}{|c|}{ Mean \pm STDEV } \\
\hline \multicolumn{3}{|c|}{$(\mathrm{mg} / \mathrm{g})$} & \multicolumn{3}{|c|}{$(\mathrm{mg} / \mathrm{g})$} \\
\hline & Cultured Sample & Dam Sample & & Cultured Sample & Dam Sample \\
\hline C12:0 & $0.213 \pm 0$ & $0.326 \pm 0$ & C14:1 & $0.244 \pm 0.001$ & ND \\
\hline C14:0 & $0.646 \pm 0.017$ & $0.391 \pm 0.006$ & $\mathrm{C} 16: 2$ & $0.849 \pm 0.029$ & ND \\
\hline C15:0 & $0.271 \pm 0.002$ & $0.341 \pm 0.001$ & C16:1 & $4.280 \pm 0.192$ & $0.201 \pm 0.004$ \\
\hline C16:0 & $3.882 \pm 0.620$ & $10.942 \pm 0.678$ & $\mathrm{y}-\mathrm{C} 18: 3$ & $0.227 \pm 0.003$ & $0.499 \pm 0.022$ \\
\hline C17:0 & $0.241 \pm 0.003$ & $0.311 \pm 0.012$ & C18:4 & $10.957 \pm 0.521$ & $1.705 \pm 0.127$ \\
\hline C18:0 & $1.157 \pm 0.050$ & $1.029 \pm 0.059$ & C18:2 & $0.545 \pm 0.019$ & $0.981 \pm 0.044$ \\
\hline C19:0 & ND & $0.306 \pm 0$ & C18:3 & $0.355 \pm 006$ & $4.943 \pm 0.382$ \\
\hline C20:0 & $0.194 \pm 0.001$ & $0.340 \pm 0.003$ & C18:1 & $1.284 \pm 0.050$ & $0.077 \pm 0$ \\
\hline $\mathrm{C} 24: 0$ & $0.353 \pm 0.009$ & ND & & & \\
\hline C26:0 & $0.488 \pm 0.093$ & ND & & & \\
\hline C28:0 & $0.277 \pm 0.030$ & ND & & & \\
\hline
\end{tabular}

Table 1: Comparison of fatty acids detected in cultured samples and those detected in samples from Vaal Dam.

Comparison of volatile organic solvent extraction (VOS) and ionic liquid extraction (IL)

Because of the importance of fatty acids compositions in nowdays research and uses of lipids the development of effective, quicker and environmentally friendly method of extraction is so important. Thus, after the study of the effect of FeSO4 concentration on the amount of fatty acids in cyanobacteria biomass using ultrasonic solid liquid extraction procedure, the technique was used to study the composition of fatty acids from cyanobacteria sampled from Vaal Dam in South Africa and its performance compared with the ionic liquid extraction technique. The results were compared to the one obtained using imidazolium based -ionic liquid extraction procedure; 3-butyl-1methyimidazolium chloride [BMIM]Cl]. It was observed that the composition of fatty acids was both qualitatively and quantitatively similar as it was confirmed statistically by one way-ANOVA to have no significant difference. This result opened up the potentiality of imidazolium based ionic liquid ([BMIM] $\mathrm{Cl}$ in particular) to be used as a quick extraction method for lipids from cyanobacteria biomass. In the comparisons of Ionic liquid (IL) extraction and volatile organic solvents (VOS) extraction procedures using the samples from Vaal Dam in South Africa, it was observed that ionic liquid extraction apart from being environmentally friendly it takes a very short time as compared to the organic solvent extraction procedure and hence suitable for handling a large number of samples. Figure 6, shows the comparison of fatty acids obtained by both ionic liquid extraction technique and volatile organic solvents extraction technique.

Thus, it was observed that the qualitative and quantitative composition of fatty acids obtained by the two extraction techniques, is more less the same, and it was confirmed statistically (one wayANOVA) that the difference between the amounts of fatty acids extracted by the two extraction techniques is not significantly different $(\mathrm{P}>0.05)$.

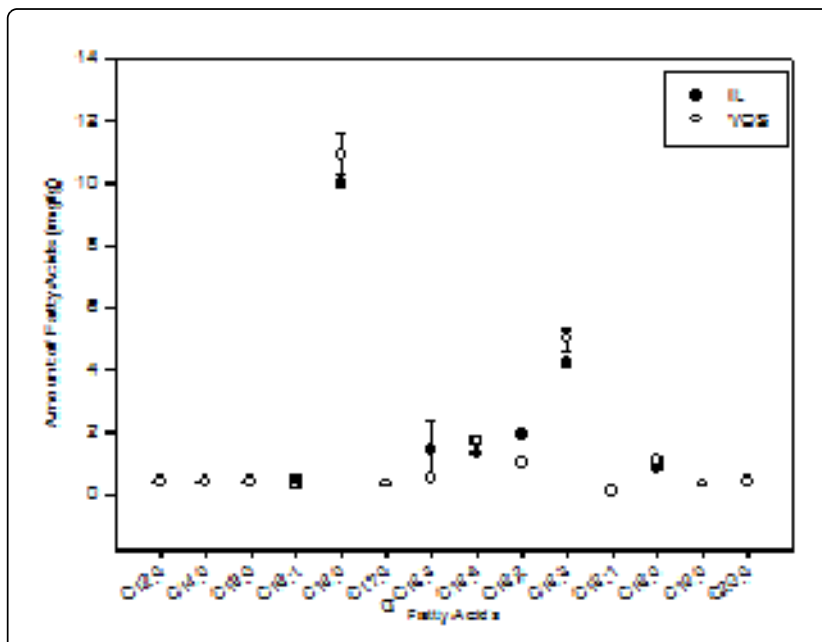

Figure 6: Comparison of fatty acids composition in the two extraction approaches; $\mathrm{IL}=$ ionic liquid extraction; $\mathrm{VOS}=$ Volatile organic solvent extraction (ie. chloroform: methanol)

\section{Conclusions}

The determined fatty acids from cyanobacteria biomass were dominated by tetradecanoic acid (C14:0), hexadecanoic acid (C16:0), 7-hexadecenoic acid (C16:1), pentadecanoic acid (C15:0), 6,9,12,15octadecatetraenoic acid (C18:4), $\gamma$-6,9,12-octadecatrienoic acid $(\gamma$ C18:3), 9,12,15-octadecatrienoic acid (C18:3), 9,12-octadecadienoic 
Citation: Kilulya KF, Bhekie B Mamba BB, Msagati TA (2015) Extraction Procedures and GCxGC-TOFMS Determination of Fatty Acids (FAs) in Cyanobacteria Cultures and the Effect of Growth Media Iron Concentration Variation on Cellular FAs Composition. J Environ Anal Toxicol S7: S7-009. doi:10.4172/2161-0525.S7-009

Page 6 of 6

acid (C18:2), 9-octadecenoic acid (C18:1) and octadecanoic acid (C18:0). The study has revealed that the variations of iron concentration in growth media affect the amount of fatty acids in the cyanobacteria biomass. The composition of fatty acids for cultured cyanobacteria contained longer carbon chain above C20 whereas the composition of cyanobacteria samples from Vaal Dam did not contain such fatty acids. From this study therefore, it is inferred that the type and amount of nutrients influence the composition of fatty acids in cyanobacteria. It was further revealed that the total amount of fatty acids in the cultured cyanobacteria decreased with increase in iron concentration. In particular it was observed that the total amount of unsaturated fatty acids decreased with increase in the concentration of FeSO 4 whereas the amount of saturated fatty acids increased with increasing concentration of $\mathrm{FeSO} 4$ and that the optimal concentration of iron was observed to be $2.2 \mathrm{mg} / \mathrm{L}$. In the comparison of the extraction techniques between the ionic liquid (IL) extraction and volatile organic solvent (VOS) extraction procedures using the samples from Vaal Dam in South Africa, it was observed that the composition of fatty acids was both qualitatively and quantitatively similar as it was confirmed statistically by one way-ANOVA to have no significant difference. Moreover, ionic liquid extraction technique takes shorter time than the volatile organic solvent extraction procedure.

\section{Acknowledgements}

The authors are thankful to the Department of Applied Chemistry of the University of Johannesburg, South Africa for research facilities and funding the research. Also the University of Dar es salaam is acknowledged for its contribution towards the success of the study.

\section{References}

1. Sharathchandra K, Rajashekhar M (2011) Total lipid and fatty acid composition in some freshwater cyanobacteria. J Algal Biomass Utln 2: 83-97.

2. Madhumathi V, Deepa P, Jeyachandran S, Manoharan C, Vijayakumar C (2011) Antimicrobial activity of cyanobacteria isolated from freshwater lake. Internat J Microbiol Res 2: 213-216.

3. Makandar MB, Bhatnagar A (2010) Biodiversity of Microalgae and Cyanobacteria from freshwater bodies of Jodhpur, Rajasthan (India). J Algal Biomass Utln 1: 54-69.

4. Senthil P, Jeyachandren S, Manoharan C, Vijayakumar S (2012) Impact of rubber industry effluent on the amino acid and fatty acid content of cyanobacteria. Eur J Exp Biol 2: 266-269.

5. Thajuddin N, Subramanian G (2005) Cyanobacterial biodiversity and potential applications in biotechnology.Current Science-Bangalore 89: 47-57.
6. Bury NR, Codd GA, Wendelaaar Bonga SE, Flik G (1998) Fatty acids from the cyanobacterium Microcystis aeruginosa with potent inhibitory effects on fish gill Na+/K+-ATPase activity. J Exp Biol 201: 81-89.

7. Ryckebosch E, Muylaert K,Foubert I(2012) Optimization of an analytical procedure for extraction of lipids from microalgae. J Am Oil Chem Soc 89: $189-198$

8. Singh SC, Sinha RP, HÃader DP (2002) Role of lipids and fatty acids in stress tolerance in cyanobacteria. Acta Protozool 41: 297 - 308.

9. Chaiklahana R, Chirasuwana N, Lohab V, Bunnag B (2008) Lipid and fatty acids extraction from the cyanobacterium Spirulina. Sci Asia 34 299-305.

10. Kusaka T, Ikeda M, Nakano H, Numajiri Y (1988) Liquid chromatography/mass spectrometry of fatty acids as their anilides. J Biochem 104: 495-497.

11. Kilulya KF, Msagati TAM, Mamba BB, Ngila JC, Bush T (2012) Study of the Fate of Lipophilic Wood Extractives During Acid Sulphite Pulping Process by Ultrasonic Solid-Liquid Extraction and Gas Chromatography Mass Spectrometry. J Wood Chem Tech 32: 253-267.

12. Thurbide KB, Hughes DM (2000) A rapid method for determining the extractives content of wood pulp. Ind Eng Chem Res 39: 3112-3115.

13. Carr AG, Mammucari R, Foster NR, (2011) A review of subcritical water as solvent and its utilisation for the processing of hydrophobic organic compounds. Chem Eng J 172: 1-17.

14. Herreroa M, Pedro J. Álvareza M,Señoránsb FJ, Cifuentesa A, et al. (2005) Optimization of accelerated solvent extraction of antioxidants from Spirulina platensis microalga. Food Chem 93: 417-423.

15. Kilulya KF, Msagati TAM, Mamba BB (2014) Ionic Liquid-Based Extraction of Fatty Acids from Blue-Green Algal Cells Enhanced by Direct Transesterification and Determination Using GC $\tilde{A}-$ GCTOFMS. Chromatographia 77: 479-486.

16. Kilulya KF, Msagati TAM, Mamba BB,Ngila JC, Bush T (2012)Determination of Lipophilic Extractives in Ionic Liquid Extracts of Eucalyptus Pulp by Gas Chromatography - Mass Spectrometry. Tanzania J Sci 38: 14-26.

17. Kim YH, Choi YK, Park J, Lee S, Yang YH, et al. (2012) Ionic liquidmediated extraction of lipids from algal biomass. Bioresour Technol 109: 312-315.

18. Kim YH, Park S,Kim MH, Choi YK, Yang YH, et al.(2013) Ultrasoundassisted extraction of lipids from Chlorella vulgaris> using [Bmim] [MeSO]. Biom Bioenerg 56: 99-103.

19. Rueter JG, Petersen RR (1987) Micronutrient effects on cyanobacterial growth and physiology. New Zeal. J. Mar. Fresh. Res. 21 : 435-445.

20. Los DA1, Murata N (1999) Responses to cold shock in cyanobacteria. J Mol Microbiol Biotechnol 1: 221-230.

21. Grobbelaar J, Botes E, Heever J, Oberholster AM, Oberholster P (2004) Toxin production by cyanobacteria. WRC Report. 